\title{
Addressing Risk Factors for Neurocognitive Decline and Alzheimer's Disease Among African Americans in the Era of Health Disparities
}

\author{
David L. Mount ${ }^{1,2}$ et al.* \\ Maya Angelou Center for Health Equity, Wake Forest School of Medicine \\ ${ }^{2}$ Founder and CEO, Mind-Body Institute of the Piedmont \\ United States of America
}

\section{Introduction}

More than any other time in the past, the world is now paying attention to the public health implications of neurological health, ranging from cognitive aging, and mild cognitive impairment to neurodegenerative conditions such Alzheimer's disease. It is estimated that every 69 seconds someone develops AD related symptoms, and by 2050, the rate of developing this condition will be about every 33 seconds (Alzheimer's Association 2011). As a leading public interest organization, the national Alzheimer's Association 2011 report also mentions that in 2010, 14.9 million family members and friends provided 17 billion hours of unpaid care to those with Alzheimer's and other types of dementia, a care valued at \$202.6 billion. Adverse neurocognitive functioning is now front and center of public health concerns as more than 5.4. million people are living with Alzheimer's disease (AD).

Within the continuum of health disparities, we are now learning that risk for Alzheimer's disease (AD) is 14-100\% more likely for African Americans when compared to Caucasian Americans (Taylor, Sloan et al. 2004; Manly 2008; Weiner 2008). For all too many African Americans, the biopsychosocial threats to brain health and neurocognitive functioning are profoundly influenced by seen and unseen factors, ranging from inequalities in education, health, neighborhoods and communities, vocational opportunities, and access to wellness promoting resources. The cumulative effect of acute and chronic inequalities may be expressed through a differentially higher burden for brain-behavior vulnerability. Hence, identifying factors that are leading to an increased risk for adverse neurocognitive health and wellbeing is of highest priority in the black community.

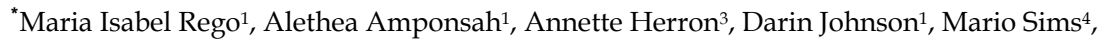
DeMarc Hickson 5 , and Sylvia A. Flack ${ }^{6}$

1 Maya Angelou Center for Health Equity, Wake Forest School of Medicine, United States of America 2 Founder and CEO, Mind-Body Institute of the Piedmont, United States of America

3 Department of Behavioral Sciences and Social Work, Winston Salem State University, United States of America 4 Department of Medicine, University of Mississippi, United States of America

5 Department of Medicine, University of Mississippi $\mathcal{E}$ Department of Epidemiology and Biostatistics, Jackson State University, United States of America 6 Center of Excellence for the Elimination of Health Disparities, Winston Salem State University United States of America
} 


\section{Public health implications of dementia related health}

Dementia is a general term used when referring to symptoms caused by disorders that affect the brain. Persons with dementia have an assortment of neurobehavioral symptoms affecting and interfering with cognitive performance, memory, personality, and activities of daily living. Alzheimer's disease (AD) is a specific type of disease process, and in the early 1990s this condition was estimated to affect 1.5 to 2 million people (Brinton 1993).

Although AD, closely followed by vascular dementia, is the more common form of dementia, African Americans are more than twice as likely to develop dementia symptoms when compared to Caucasian Americans (Alzheimer's Association 2002; Demirovic, Prineas et al. 2003; Fitzpatrick, Kuller et al. 2004; Bennett 2007; Plassman, Langa et al. 2007; Fitzpatrick, Kuller et al. 2009). For over a decade, the prevalence of dementia in the African American community has been characterized as a "silent epidemic". Issues of accessibility to medical care have been linked to the prevalence of dementia in the black community (Alzheimer's Association 2002). Chances are, African Americans may receive critical neurological attention too late and miss the window for therapeutic interventions. This is particularly concerning given the growing discussion about reversal of adverse neurocognitive functioning, including dementia (Arendash, Mori et al. 2009; Etgen, Sander et al. 2010; Mangialasche, Solomon et al. 2010). Moreover, African-Americans have a higher rate of vascular disease such as T2DM, a condition thought to increase brain dysfunction in higher cortical abilities. Despite the significance of an early diagnosis of Alzheimer's disease (AD), African Americans are diagnosed in later stages of the disease, demonstrate greater cognitive impairment at the time of diagnosis, and receive less adequate treatment following diagnosis (Hughes, Tyler et al. 2009; Chin, Negash et al. 2011). As the major public health emphasis for African Americans remains focused around physical disease (e.g., heart disease, lower limb amputations, and kidney disease), there is growing concern that health care professional and the patients for whom they provide care may be missing opportunities for connecting integrative mind-body health.

Although the exact cause of Alzheimer's is not completely understood, experts have recently identified a genetic and physiological mechanism involving the insufficient breakdown and recycling of amyloid protein in the brain. This action process leads to cell death and weakened neuronal communication. Guidelines identifying and classifying individuals with possible, probable, and definite AD were established through a joint effort supported by the National Institute of Neurological and Communicative Disorders and Stroke (NINCDS) and the Alzheimer's Disease and Related Disorders Association (ADRDA) (McKhann, Drachman et al. 1984). Multi-center studies have shown these guidelines to have adequate sensitivity (83\%) and specificity (84\%) (Blacker, Albert et al. 1994). Additional diagnostic criteria for probable $\mathrm{AD}$ include medical examination, deficits in two or more neurocognitive domains, progressive worsening of memory and other neurocognitive domains, no disturbance of consciousness, and absence of systemic disorders or other brain diseases that could be the cause of decline.

\section{Type 2 diabetes immediate and intermediate crises}

T2DM is a blood sugar dysregulation disorder associated with insulin resistance, insulin deficiency, or both. Individuals can remain undiagnosed for as long as seven years (Harris, Klein et al. 1992). T2DM is a costly, life-threatening chronic disease that is associated with a 
high degree of medical complications and an estimated price tag of $\$ 100$ billion a year (O'Connor 2006). This metabolically driven chronic illness is at an all time high in black America, and tied primarily to health behaviors that include poor dietary habits and sedentary lifestyles (Bell, Bertoni et al. 2010). In addition to physical health markers, persons living with diabetes are known to live with an elevated risk of developing adverse neurocognitive problems, ranging from mild cognitive impairment to more severe brain health problems including AD related dementia. Memory related problems were initially reported, particularly among persons with complications of peripheral neuropathy and/or elevated A1c levels (Perlmuter et al., 1984). These findings have been substantiated through meta-analysis research methodology (Strachan, Deary et al. 1997). At this, based upon research published between 1980 and 1995, Strachan and colleagues found that there was a connection between history of T2DM and verbal memory dysfunction. Supplementing these findings, multi-site longitudinal studies including, the Rotterdam Study (Ott, Stolk et al. 1996), the Honolulu-Asia Aging Study (Peila, Rodriguez et al. 2002), and the Epidemiology of Vascular Aging Study (Fontbonne, Berr et al. 2001) have also found evidence of a diabetes-neurocognitive functioning compromise. When studying younger Type 2 diabetes cohorts, non-age related changes in information processing speed are observed (Ryan and Geckle 2000). As noted in their published findings from the Atherosclerosis Risk in Communities cohort, a large biracial, multisite, longitudinal investigation of initially middleaged individuals, (Knopman, Boland et al. 2001) observed that persons with Type 2 diabetes demonstrated problems with language skills tasks.

The stated inconsistencies in the area of diabetes and brain may reflect differences in how well researchers have characterized the population. Toward achieving a more standardized covariate modeling of the data, some relevant markers need mentioning. Because the community of persons living with T2DM is not homogenous, it is important to explore the range of biopsychosocial candidate markers implicated in a loosely defined Diabetesneurocognitive risk paradigm. Duration of diabetes is identified as a risk marker. For instance, when persons with well controlled diabetes are compared with relatively healthy individuals without diabetes, there are relatively no discernable differences on behavioral tasks measuring verbal memory, except when duration of diabetes is considered (Cosway, Strachan et al. 2001). Duration of diabetes as a brain-cognition threshold marker has been previously reported in the literature (Stewart and Liolitsa 1999; Logroscino, Kang et al. 2004).

Insulin resistance is a physiological condition where the natural hormone, insulin, becomes less effective at lowering blood sugars. Insulin resistance as also been addressed has an inflammatory process (Shoelson, Lee et al. 2006). The poor supply of glucose to the brain can also cause neurological symptoms such as: drowsiness, confusion, loss of consciousness, seizures, and permanent brain damage. Elevated blood glucose levels can damage small blood vessels (miscrovascular disease) that in turn affect the eyes (diabetic retinopathy), kidneys (nephropathy), and nerve damage (i.e.,neuropathy). Elevated blood sugar or hyperglycemia is a significant complication experienced in Type 2 diabetes. Elevated blood sugar is shown to decrease acetylcholine synthesis in rat brain (Squadrito, Trimarchi et al. 1986; Welsh and Wecker 1991). Acetylcholine is a neurotransmitter that appears to be involved in learning and memory, and is severely diminished in the brains of persons with Alzheimer's disease. Insulin resistance, as inflammatory response, may lead to a situation of brain cells losing their ability to convert glucose to energy, and begin to starve.

There is reason to believe that dementia, in the context of T2DM, is driven through a stress pathway and inflammatory sensitive process. Stressful situations repeatedly activate 
Allostatic load responses leading to a wear and tear of the body (McEwen and Stellar 1993). This cumulative wear and tear or Allostatic load represents a model of how psychosocial factors impact the body and lead to disease (Nielsen, Seeman et al. 2007). There seems to be three stages of allostasis. During the primary stage the body releases stress hormone molecules and their antagonists as well as anti-inflammatory cytokines which are the Allostatic load biomarkers called primary mediators (Juster, McEwen et al. 2010). These molecules' synergistic effects impact cellular levels activities which compromise the integrity of Allostatic mechanisms (Juster, McEwen et al. 2010). In order to compensate for unbalanced production of these primary mediators, subsidiary biological systems change their operating ranges to maintain functions at chemical, tissue and organ levels (Juster, McEwen et al. 2010). At a secondary stage, also called prodromal stage, metabolic, cardiovascular and immune parameters arrive at sub-clinical levels (Juster, McEwen et al. 2010). The third and final stage or the Allostatic overload is when physiological dysregulation leads to tertiary outcomes or disease (Juster, McEwen et al. 2010). Even more dangerous are brain changes that are associated with chronic stress and allostasis since it further reduces our abilities to cognitively process and physiologically respond to stressors (Juster, McEwen et al. 2010). Therefore, this model explains how the dysregulation of systems that are supposed to balance our bodies through environmental demands serves as a key mediator for increased disease risk (Nielsen, Seeman et al. 2007).

Several, serum markers of inflammation have been speculated to burn neurocognitive abilities. Findings from the Health, Aging, and Body Composition Study demonstrated that serum markers of inflammation, especially IL-6 and CRP, were prospectively associated with cognitive decline in well-functioning African-American and white elders, as measured by the Modified Mini-Mental State Examination (3MS)(Yaffe, Lindquist et al. 2003). Creactive protein (CRP), a marker of inflammation, is generated at a higher level in obese people, and mild elevation in CRP increases risk of heart attacks, strokes, high blood pressure, muscle weakness and fragility. In a more recent cognitive aging related study (Noble, Manly et al. 2010), findings showed that participants in the highest high-sensitivity (hs) CRP tertile had higher adjusted odds of impaired memory (odds ratio [OR], 1.5; 95\% confidence interval $[\mathrm{CI}], 1.0-2.1 ; P=.03$ ) than participants in the lowest tertile. Subjects in the highest hs CRP tertile also had greater odds of visuospatial impairment (OR, 1.6; 95\% CI, 1.02.3; $P=.03$ ). Higher hsCRP was not associated with executive or language impairment. Persons with at least 1 APOE $\varepsilon 4$ allele and hsCRP in the highest tertile had the greatest odds of impaired memory (OR, 2.7; 95\% CI, 1.6-4.4). While CRP is associated with body mass index, the evidence on the association between BMI and cognitive functioning in aging research is mixed. For instance, in one biracial community population aged 65 and older, BMI was not predictive of cognitive decline in a cognitively unimpaired community population (Sturman, de Leon et al. 2008); however, results from a Swedish community population study, published in the Journal of Gerontology (Dahl, Hassing et al. 2010), found that higher midlife BMI scores precede lower general cognitive ability and steeper cognitive decline in both men and women. Yet, data from the Cardiovascular Health Study (Fitzpatrick, Kuller et al. 2009) seems to have indicated no association indicated no association of BMI with greater dementia risk.

As diabetes is a constellation of signs and symptoms, a salient feature of poor glycemic control is increased risk for atherosclerosis. Atherosclerosis, a disease of vessel wall thickness and plaques of the carotid arteries, is a risk factor for non-age related changes in brain functioning (Hofman, Ott et al. 1997) and a consequence of several lifestyle factors 
(e.g., cigarette smoking, sedentary lifestyle, obesity). Reporting on their findings from the Rotterdam Study, Hofman and associates (year) found that atherosclerosis is associated with dementia and the odds ratio for Alzheimer's disease in those with severe atherosclerosis compared with those without atherosclerosis was 3.0 (95\%, CI 1.5-6.0; p = 0.001).

Similar to atherosclerosis, hypertension is shown to alter brain functions (Kilander, Nyman et al. 1998; den Heijer, Launer et al. 2005). Variables that may alter (i.e., moderate) the relationship of hypertension to brain functioning include age, education, several characteristics of elevated blood pressure or hypertension, and the presence of concurrent diseases (Waldstein 2003). The data suggests that while hypertension is a risk factor for cerebrovascular health independent of Type 2 diabetes, the joint contributions of both diseases elevate stroke mortality $(\mathrm{Hu}$, Sarti et al. 2005). Pathologies of the peripheral vasculature are responsible for a wide variety of clinical conditions, premature disability, and early death. With these and other factors in mind, T2DM increases risk for peripheral arterial disease (PAD), a common disease affecting 8 to 12 million Americans (Selvin and Erlinger 2004) Occurring in about $8 \%$ of African Americans, $5 \%$ of Mexican Americans, and about 3\% of Caucasian Americans(Rucker-Whitaker, Greenland et al. 2004; Nelson, Reiber et al. 2007; Meadows, Bhatt et al. 2009). PAD occurs when a fatty material called plaque builds up on the inside walls of the arteries that carry blood from the heart to the head, internal organs, and limbs. Peripheral arterial disease (PAD) is a manifestation of systemic atherosclerosis, is associated with significant morbidity and mortality, and is an important marker of subclinical coronary heart disease, and stroke (Selvin and Erlinger 2004; Khattab, Ali et al. 2005). PAD prevalence increases dramatically with age and disproportionately affects persons of color (Collins, Petersen et al. 2003; Selvin and Erlinger 2004), and the comorbidity of PAD and Type 2 diabetes greatly increases mortality (Leibson, Ransom et al. 2004). Three types of studies on the investigation of cognitive functioning have emerged including PAD severity compared to healthy controls (Waldstein, Tankard et al. 2003), asymptomatic PAD and inflammatory process (Mangiafico, Sarnataro et al. 2006), and PAD and coronary heart disease with stroke (Phillips and Mate-Kole 1997; Rao, Jackson et al. 1999); (Mukherjee, Eagle et al. 2007). PAD can impair physical health and diminish the ability to walk since physical activity is associated with oxygenated blood flow to brain and cognitive agility (Yaffe, Barnes et al. 2001; Allmer 2005; Singh-Manoux, Hillsdon et al. 2005). Jointly, poor circulation in the legs can be associated with poor circulation in the heart and in the brain. Such questions would concern how and to what extent persons with T2DM develop their continuum of risk for adverse brain health and neurocognitive dysfunction, including neurodegenerative disease, such as dementia of the AD type. Recent clinical data show that rates of diabetes and other cardiovascular disease health problems are increasing among African Americans in their middle-ages and younger (Krishnan, Cozier et al. 2010; Cali and Caprio 2008; Steinberger, Daniels et al. 2009). An earlier onset of physical health problems correlated with accelerated brain aging will likely translate into a greater burden of neurocognitive problems in younger cohorts. To that extent, blood circulation components, including volume, flow, access, and nutrition quality may prove beneficial in elucidating the upward-bound body-brain functionality and dynamics central to critical psychoendoneuroimmunology questions.

For the most part, T2DM does not appear to have immediate effects on brain health and cognitive functioning. All things being considered, it is equally plausible that adverse neurocognitive risk and event is the summed expression of an inverse neurocognitive resilience-inflammatory response process. That is, as one's resilience is diminished there 
might be an appreciable spike in inflammatory activity. Risk for brain health complications along the diabetes-brain connectivity spectrum is more characteristic of intermediate effects, especially when there is evidence of diabetes being poorly controlled. This said, a general saying in clinical neuropsychology is "its not just the brain it happens to; its whose brain it happens to". Such a statement warrants attention to acknowledging the absence of a socialecological perspective in addressing prevention and treatment, as well as the formulation of intervention efforts in taking serious action to eliminate the burden of dementia related disparities faced by African Americans, particularly those suffering from vascular diseases.

\section{Built environment and social determinants of $A D$ risk}

The built environment in the context of health can provide insight into how African Americans may experience a differentially high risk for dementia. The term built environment is used to signal human-made structures and how those environmental components come to shape our health, whether we are talking about neighborhood-level factors or more broad ranging elements that constitute community and its accessible and inaccessible resources. Within the built environment lies a host of factors operating in concert, but are there clues and solutions in the built environment that could be used to reposition translational research on brain and neurocognitive impairment research? As some brain functions are more sensitive to aging effects than others, neurobiological and contextual factors such as environmental demands and cultural experiences independently and simultaneously influence underlying neural circuits that activate and deactivate cognitive processes (Salthouse 1996; Baltes, Staudinger et al. 1999; Von Dras and Blumenthal 2000; Park and Gutchess 2006). Environmental factors shown to affect health include housing and indoor air quality (McEwen 2008; Lan and Lian 2009; van Hoof, Kort et al. 2010), neighborhood location, noise, crowding, socioeconomic status (Glass, Bandeen-Roche et al. 2009; Hackman and Farah 2009; Van Gerven, Vos et al. 2009; Yen, Michael et al. 2009), access to grocery stores, nutritious foods (Gomez-Pinilla 2008; Rose 2010), and space and safety forphysical activity (Kramer and Erickson 2007; Hillman, Erickson et al. 2008; Lautenschlager, Cox et al. 2008; Scarmeas, Luchsinger et al. 2009).

It is generally accepted that chronic stress and chronic physical diseases accelerate physiological changes in multiple organ systems independent of age (Sieck 2003; Roth, Mattison et al. 2004). As psychosocial markers, negative emotional functioning is problematic for achieving optimal brain health. That is, emotional instability stimulates the release of various hormones, alters metabolic activity, and is linked to risk for elevated blood glucose levels. Hostility and impatience influence body physiological in the direction of blood pressure elevations (Yan, Liu et al. 2003). Negative emotional states and both acute and chronic social stressors increase cardiovascular symptoms (Ferketich, Schwartzbaum et al. 2000; Everson-Rose and Lewis 2005). In postmenopausal women, the evidence suggests that perceived chronic stress was associated with decreased grey matter volume in the right orbitofrontal cortex and right hippocampus (Gianaros, Jennings et al. 2007). Studies have shown that chronic stressful life events (e.g., perceived discrimination or chronic burden) may impair the sympathetic nervous system, as well as neuroendocrine stability, cognitive and emotional functions that the hippocampus supports: spatial information, regulating the HPA axis and processing the contextual aspects of emotional events.(Krieger and Sidney 1996; Sapolsky 2003; Conrad 2006; Aldo Ferrara, Guida et al. 2007; Cohen, Janicki-Deverts et al. 2007; Albert, Ravenell et al. 2008). 
For sometime now, researchers have found that chronic stress derails the body's physiology, including the brain's corticotropin-releasing factor, interleukin-6, brain-derived neurotrophic factor, and insulin-like growth factor-1(Bonne, Gill et al. 2010), and has a consequential impact on AD risk (Nakajima, Ohsawa et al. 2010; Gasparini and Xu 2003; Araki, Kume et al. 2009). To the point, chronic stress can diffuse neural signaling, diminish the cells ability to cope with adversity and potentially trigger accelerated age-related damage to the hippocampus. The adrenal glucocorticoids are thought to be responsible for this damage given its ability to compromise energy metabolism and make neurons more vulnerable to glutamate excitotoxicity (Smith 1996; Mattson, Maudsley et al. 2004). Hence, stress has been found to decrease brain-derived neurotrophic activity in the hippocampus and other possible brain areas. The term disrupted energy in the context of metabolism is being used more frequently in brain and neurocognition research. The pathogenesis of neuronal circuit dysfunction connects to overwhelmed neurocognitive compensatory mechanisms and is speculated to result from perturbation in cellular energy metabolism, level excitation or inhibition and neurotrophic factor release (Kapogiannis and Mattson 2011).

As a chronic stress psychosocial marker, socioeconomic status (SES) has received increasing attention as an explanatory mechanism for health disparities. SES and its associated proxy indicators (i.e. income, employment, education, housing, social cohesion, life expectancy, etc.) are thought to negatively affect healthcare utilization and health outcomes, including premature death (Gostin et al., 2004; McGennis, Williams-Russo, \& Knickman, 2002). A number of studies have indicated that African Americans are at greater risk for suffering from chronic disease and disease morbidity often operating through injustice and discrimination (Clark, 2000; Kessler et al., 1999, Williams et al., 1997). Chronic stress reduces an individual's functional capacity, and has an untoward effect on sustainable healthy functioning. The "isms" that impact health outcomes (i.e. racism, institutionalism, ageism, sexism, etc.) increase mortality rates, as discrimination is inversely related to medical wellbeing, e.g. cardiovascular disease, (Krieger \& Sidney, 1996), as well as emotional health (Branscombe, Schmitt, \& Harvey, 1999; Fischer \& Shaw, 1999).

A last and central component of the built-environment is access to and quality of caregiving on neurocognitive wellbeing. The role of African American caregiver in the case of caring for someone with adverse mental functioning is loosely addressed in the dementia literature. In the Black community caregiving occurs at several levels. Within the context of social services, caregiving is defined as providing assistance or care to someone who is unable to perform some or all Activities of Daily Living (ADLs) and/or Instrumental Activities of Daily Living (IADLs). Examples of ADLs are bathing, eating (unable to feed oneself), and dressing. IADLs are complex activities such as driving, planning, and cooking. There is some evidence suggesting African American couples have others to assist them with ADLs compared to Caucasian couples who only have themselves to rely on (Feld, Dunkle, and Schroepfer ,2005; Yarry, Stevens, McCallum, 2007).

In 2009, approximately 10.9 million family caregivers in the United States did not receive payment for the care they provided to a family member with Alzheimer's disease (2010 Alzheimer's Association Report). An informal caregiver is someone who is not being compensated monetarily for the care they provide. An informal caregiver can be a family member (e.g. husband, cousin, extended family member), a neighbor, or a friend. Burnout is potentially a caregiving risk factor as African American caregivers are less likely to utilize formal care such as respite care or nursing homes services (Fiscella, Franks, Gold, \& Clancy, 2000; Cagney \& Agree, 1999; Murtaugh, Kemper, Spillman, \& Carlson, 1997; Belgrave \& 
Bradsher, 1994. Despite of the declining health of the elderly caregiver, they are more likely to be the caregiver and care recipient ((Yarry, Stevens, McCallum, 2007; Sorensen and Pinquart, 2005; Hooyman and Kiyak,1996). In North Carolina, the number of Alzheimer's/dementia caregivers was 311,578 in 2007 but rose to 356,851 in 2009. The 2007 group provided 268,953,971 hours of unpaid care that year at an economic value of $\$ 2,845,533,016$. The 2009 group provided $406,381,406$ hours of unpaid care that year at an economic value of $\$ 4,673,386,174$. For South Carolina, the number of Alzheimer's/dementia caregivers was 159,221 . They provided $137,439,556$ hours of unpaid care that year, which had an economic value of $\$ 1,454,110,507$ (Alzheimer's Association, 2008).

Although not conclusive, there is strong evidence that African Americans experience less caregiving depression compared with Caucasian Americans. (McCallum, Longmire, Knight, 2007; Sorensen \& Pinquart, 2005; Plant \& Sach-Ericsson, 2004; Dilworth-Anderson, Williams, Gibson, 2002; Knight, Silverstein, McCallum, \& Fox, 2000; Cox, 1995; Allen, 1993). The church environment has been shown to serve as a potential emotional buffer among this population(Hines-Martin, 1998). Religion has been found to be a significant coping mechanism for African American informal caregivers. Caregivers who attend or are active members in a church report less experience of depression and stress (Sorensen \& Pinquart, 2005; Coon et al., 2004; Haley et al, 2004; Stuckey, 2001; Hines-Martin, 1998; Wood \& Parham, 1990). More than likely, the church buffering effect may be acting in concert with what has been termed the mutual aid system (Dilworth-Anderson, 1992; Wood \& Parham, 1990). The mutual aid system is the extended family which included the parents, children, uncles and aunts, grandparents, close friends, and the community. Specifically, one study investigating the kin support and use of formal caregivers (i.e. licensed or certified professionals such as nurses, home aids, etc.) found that African American caregivers who are caring for an impaired elderly family member had a larger number of "nuclear kin" available to provide assistance compared to Caucasian caregivers (Sterritt, Pokorny, 1998). It has been further observed that African Americans caregivers are less likely to be spouses, and more likely to be extended family members (Dilworth-Anderson et al., 2002; Knight \& McCallum, 1998).

\section{Opportunities for promoting and restoring neurocognitive health}

Restoration is generally thought of as involving an integrative renovator-rejuvenating dynamic embodying a holistic reserve necessary to achieving optimal physical, mental, and emotional health functioning. However, the pathways of restoration and reversal of adverse neurocognitive health remain poorly understood. There is growing speculation that increasing cognitive stimulation, consuming more fruit and vegetables, increasing physical activity, maintaining emotional balance, and reducing diabetes and its health related complications would have a noteworthy public health impact on reducing the incidence of dementia, outweighing even the effects of removing the principal known genetic risk factor (Ritchie, Carriere et al. 2010). As a component of the built environment, social network engagement has been described in terms of its brain health benefits. For instance, social network research suggests that social engagement surrounding mental, social, productive, and leisure activities appears to have an inverse relationship to dementia incidence (Amieva, Stoykova et al. 2010; Wang, Karp et al. 2002; Akbaraly, Portet et al. 2009; Hughes, Chang et al. 2010).

Caffeine's role in cognitive impairment research provides some preliminary insights. For instance, in the basic sciences, AD transgenic mice given a moderate level of caffeine intake 
have been shown to experience a memory restoration and reversal of AD pathology, suggesting the treatment potential of caffeine in cases of established AD (Arendash, Mori et al. 2009). From human-level research, there are clinical findings demonstrating the association between caffeine consumption and lower cognitive change over time to be statistically significant for women only and dose-dependent (Ritchie, Artero et al. 2010).

The impact of physical activity, nutrition and dietary quality on the aging central nervous system has multiple implications for African Americans neurocognitive health.

There is increasing evidence that physical activity and diet are equally beneficial in reducing risk for AD (Scarmeas, Luchsinger et al. 2009). Results from the Cardiovascular Health Cognition study ( $\mathrm{N}=299$; mean age 78 years) show that greater amounts of walking related physical activity was related with greater gray matter volume, which is associated with protection from neurocognitive impairment(Erickson, Raji et al. 2010). Although typically treated as a separate component of health and wellbeing, diet and, nutrition are as relevant to deconstructing the neurological consequences of the built-environment as SES, chronic stress, and other psychosocial markers. As one of the Omega-3 fatty acids, DHA is considered one of the primary structural fatty acids in brain gray matter, influencing and promoting communication between brain cells.. Specifically, dietary intake of omega-3 fatty acids and weekly consumption of fish may improve visual, mental, cardiovascular health and reduce the risk of incident Alzheimer disease (Morris et al., 2003.; Mattson, 2004; Arterburn, 2006). In persons characterized with mild to moderate AD from the randomized double blind OmegAD trial, researchers found that administration of $\omega-3$ fatty acid did not delay the rate of cognitive decline according to the MMSE or the cognitive portion of the Alzheimer Disease Assessment Scale. However, positive effects were observed in a small group of patients with very mild AD (MMSE > 27 points) (Freund-Levi et al., 2006).

Data from the National Health and Examination Survey III examining the association of race, education, and dietary intake, showed that while education attainment was positively related to intake of calcium, magnesium, and potassium among whites, African Americans consumed less calcium, magnesium, and potassium regardless of educational achievement (Ford, 1998). As a function of diet, potassium supplementation has been studied on its effects to the blood pressure of African Americans, showing positive results in substantially reducing blood pressure in persons with low potassium consumption (Brancati et al., 1996; Appel et al., 2006). In a more recent study by Individuals and the National Health and Nutrition Examination Survey, utilizing the Continuing Survey of Food Intakes questionnaire, investigations revealed similar findings that African Americans in all age groups consume fewer mean servings of total dairy, milk, cheese, and yogurt per day than non-African Americans, and have lower mean intakes of calcium, magnesium, and phosphorus (Fulgoni et al., 2007). Among African Americans, the intake of fruits and vegetables, as well as food store characteristics, store location and perceptions of the selection/quality and affordability of fresh produce mediate perceived nutritional options, at the community level (Zenk et al., 2005;). Elsewhere, African Americans' higher frequency of eating at fast-food restaurants was positively associated with fair/poor self-rated health, weak belief in a diet-cancer relationship, low self-efficacy for healthy eating, weight dissatisfaction, and perceived difficulties of preparing healthy meals and order healthy foods in restaurants (Satia et al., 2004). At last, the issues of nutrition and dietary selfmanagement represent a major piece of neurocognition health and the brain protection campaign. This is largely speculated from basic science studies, clinic-base trials, and cognition related epidemiological studies observing that higher dietary intake of 
antioxidants, vitamins B6, B12,folate, unsaturated fatty acids and fish are related to greater likelihood of neurocognitive protection reducing risk for untoward neurocognitive functioning, including AD (Burgener, Buettner et al. 2008; van der Beek and Kamphuis 2008; Carrie 2009; Dangour, Whitehouse et al. 2010).

T2DM is almost always preceded by obesity. Obesity, eating behavior, and physical activity are also associated with multiple behavior changes including brain-behavior functioning. As the body of evidence linking nutrition and dietary behavior to incidence of neurocognitive illnesses increases, community-based researchers and practitioners will need to translate this knowledge into procedures and strategies to advance African American neurocognition risk prevention research. Such community-level interventions will want to be mindful of the need to better understand the barriers to consuming specific dairy products at all levels of African American communities (e.g., SES, education attainment, gender, urban vs. rural locations). While diet and nutrition may be important modifiable risk factors in preventing adverse neurocognitive disease, conclusive evidence has yet to emerge. (Coley, Andrieu et al. 2008).

In short, whether brain and its supporting abilities can be restored is stimulating debate across scientific fields. At the heart of this debate is a gene-environment that may actually represent the subcomponents of where you live, what you experience, how you feel, and how you relax. Stated slightly different, neurocognitive aging may be a function of built-environment-brain energy balancing operating on some biopsychosocial continuum across time.

\section{Conclusion}

\subsection{Challenges, opportunities, and implications}

Pathologies of the central nervous system and peripheral vasculature are responsible for a wide variety of clinical conditions, premature disability, and early death. T2DM is a diet, energy, nutrition, health behavior, and lifestyle driven condition. Brain health protection and promotion are equally responsive to diet, energy, nutrition, health behavior, and lifestyle components. Several nutritional factors can influence mental health, including: overall energy intake, intake of the energy-containing nutrients (proteins, carbohydrates, and fats), alcohol intake, and intake of vitamins and minerals. The downstream occurrence of elevated risk for acquired brain changes in Americans African is inextricably linked to the upstream chronic diseases strongly tied to the social determinants of health. The social determinants of neurocognitive impairment link to physical health conditions such as high blood pressure, diabetes, heart disease, stroke, and psychiatric health conditions such as chronic depression and excessive psychological distress, as well as social resource deprivation, all known risk factors for neurocognitive illness. The built environment in the context of health can provide insights about how African Americans may experience a differentially high risk for dementia. In turn, the socio-environment plays a major role from brain development, brain stimulation to brain nutrition, brain trauma and brain aging.

The bourgeoning research underscores that there exists multiple explanations for why Africans Americans are at higher risk for preventable neurocognitive illness. There appears to be consistent findings that social factors (e.g., discrimination, biased access to resources, environmental social injustice) are a major deterrent to sustainable neurocognitive quality of life. For T2DM, untoward neurobehavioral changes will negatively impact quality-of-life and self-care management, which could place African Americans living with chronic health 
complications at additional disadvantage for underperforming in their daily physical disease management regimen, including adhering to medical regimen, scheduling appointments, health knowledge, health service utilization behavior, and prevention of secondary diseases. The factors contributing to psychosocial wellbeing may be important predictors of decreasing brain-behavioral status and might partly be related to experiences with racial discrimination. Understanding neurocognitive variability among persons living with diabetes, their activities of daily living, as well as their perceived quality of life requires a commitment to work across conventional disciplinary boundaries. Showing the relationship of psychosocial factors to differential risk for brain-behavior connectivity in a well characterized population will serve to help clinicians identify adults at high-risk for non-age related brain and cognitive changes and those who may be at additional high risk for self-management difficulties that could lead to stroke and/or dementia. Figure I, below, can be utilized to inform other researchers about these translational research efforts.

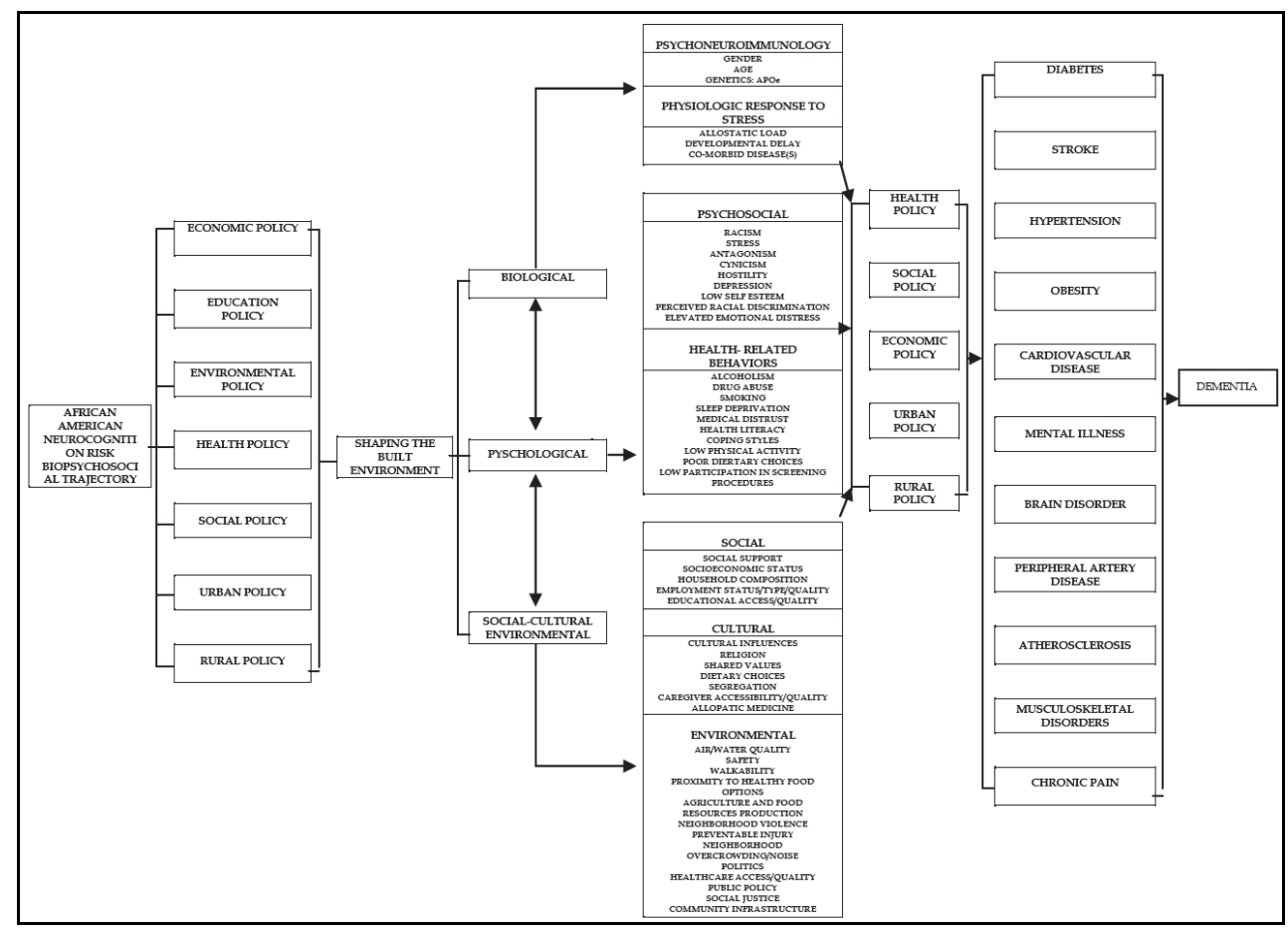

Fig. 1. Alzheimer's disease risk as a biopsychosocial determinant of health

One of the most challenging health care issues facing African Americans and the United States as a whole is the rising rates of dementia. Dementia prevention is one of the most critical health disparity agenda items of the $21^{\text {st }}$ century. Dementia not only compromises the patient, it affects the family, the church, the neighborhood, the community, the workforce, the healthcare system, as well as the public policy programs. From this viewpoint, no one can afford to ignore the need to develop programs and interventions that prevent, delay, and/or eliminate the public health burden of dementia. Our research team has planned, developed, and are working toward implementing several programs. 


\subsection{Neurocognitive health screening and monitoring in primary care}

While African Americans may have low access to specialty care, developing state-of-the-art primary care screening, evaluation, and monitoring will prove necessary in order to address dementia risk in the black community. This said, important gaps remain in our knowledge about screening for and early treatment of dementia. There are several benefits to early detection of dementia (e.g., individual and family planning, care coordination); however, there is a need for determining and examining the evidence that screening serves as an added value in the context of patient care. More attention is needed centered around identifying promising brief screening tools that can be implemented in primary care settings.

\subsection{Participatory community-based AD awareness promotion}

Developing drug therapies are essential, but drugs will not achieve therapeutic intent if patients are not willing to take them as prescribed. Our research team is focused on developing, testing, and implementing community outreach and health promotion. This work addresses the underrepresentation of African Americans in dementia related clinical research in urban and rural communities. Some of our objectives include identifying barriers to recruitment and addressing factors associated with lower participation rates in clinical research. The use of community-based participatory engagement as a strategy for addressing barriers to recruitment and retention is at the heart of our work.

In conclusion, because of a possible dementia epidemic, focusing narrowly on susceptibility genes and identifying genetic, clinical, and neuropathological subtypes of AD may miss the larger story in the context of upstream factors and cognitive health in black America. The critical question seems to be what factors are setting African Americans up on this downward neurocognition slope? With an eye toward protection, prevention, and neurocognitive promotion, we must start to unpack the biopsychosocial dimension of health that influences risk for adverse neurocognitive wellbeing and that provides clues to potential risk factor modification strategies among African Americans.

At this point in the field of neurocognitive health research and public health protection and promotion, researchers have not been able to pin down or know exactly what causes Alzheimer's disease. But, the accumulating body of research in the chronic physical disease literature is motivating brain researchers to consider mind-body explanations in understanding risk for dementia related conditions. Threats to brain health appear to be elevated in the presence of Type 2 diabetes and its associated health complications, including high blood pressure, high cholesterol, lack of exercise, malnutrition and psychological distress. This chapter has identified and characterized potential modifiable risk factors for adverse neurocognitive health in the context of African Americans diagnosed with T2DM.

This chapter should have advanced insights about the strategies that could promote awareness of AD risk and health care outcomes among African Americans with T2DM. Compared to the amount of public health attention focusing on African Americans and T2DM, little attention has focused on treating T2DM among African Americans as a neurodegenerative primary prevention public health concern. Moving forward, it will be critical to ensure that we clearly understand the lay health perspectives as it relates to meaning, health beliefs, and socio-ecological determinants attached to risk for neurocognitive impairment and $\mathrm{AD}$ within and across the black community. If African Americans continue to receive little education on neurocognitive risk factors and health 
promotion, continue to develop chronic diseases that threaten brain health at earlier ages, continue to experience psychosocial trauma, and continue to experience problems obtaining early intervention for changes in neurocognitive functioning, the possibility that neurocognitive illness will disrupt this community remains very high. The information regarding functions protecting, promoting, and interfering with neurocognitive health lag further behind information on cancer, heart disease, and diabetes. Health conditions that were once considered age-related are now being diagnosed in young and middle adulthood. This heightens the public health urgency for why funding supporting research, treatment, education, and community outreach addressing African American neurocognition health is the new civil rights issue of the $21^{\text {st }}$ century.

\section{References}

Akbaraly, T. N., F. Portet, et al. (2009). "Leisure activities and the risk of dementia in the elderly." Neurology 73(11): 854-861.

Albert, M. A., J. Ravenell, et al. (2008). "Cardiovascular risk indicators and perceived race/ethnic discrimination in the Dallas Heart Study." Am Heart J 156(6): 1103-9.

Aldo Ferrara, L., L. Guida, et al. (2007). "Blood pressure at rest, during $24 \mathrm{~h}$ monitoring and in response to sympathetic stimulation in hypertensive patients with metabolic syndrome." Int J Cardiol 117(3): 312-6.

Allmer, H. (2005). "Physical activity and cognitive functioning in aging." Journal of Public Health 13(4): 185-188.

Alzheimer's Association (2002). "Alzheimer's Association, African Americans and Alzheimer's disease: The silent epidemic, Alzheimer's Association, Chicago (2002).

Alzheimer's Association (2011). "North Carolina Alzheimer's Statistics, Report retrieved in March 2011 from ".

Amieva, H., R. Stoykova, et al. (2010). "What Aspects of Social Network Are Protective for Dementia? Not the Quantity But the Quality of Social Interactions Is Protective Up to 15 Years Later." Psychosom Med 72(9): 905-911.

Araki, W., H. Kume, et al. (2009). "IGF-1 promotes [beta]-amyloid production by a secretaseindependent mechanism." Biochemical and Biophysical Research Communications 380(1): 111-114.

Arendash, G. W., T. Mori, et al. (2009). "Caffeine Reverses Cognitive Impairment and Decreases Brain Amyloid- $\beta$ Levels in Aged Alzheimer's Disease Mice." Journal of Alzheimer's Disease 17(3): 661-680.

Arendash, G. W., T. Mori, et al. (2009). "Caffeine reverses cognitive impairment and decreases brain amyloid-beta levels in aged Alzheimer's disease mice." J Alzheimers Dis 17(3): 661-80.

Baltes, P. B., U. M. Staudinger, et al. (1999). "Lifespan psychology: theory and application to intellectual functioning." Annu Rev Psychol 50: 471-507.

Bell, R., A. Bertoni, et al. (2010). The epidemiology of diabetes in African Americans Diabetes in Black America: public health and clinical solutions to a national crisis. F. V. Jack L Jr, Graham GN, eds. Roscoe (IL), Hilton Publishing Company: 5-25

Bennett, D. A. (2007). "Editorial comment on 'Prevalence of dementia in the United States: The aging, demographics, and memory study' by Plassman et al." Neuroepidemiology 29(1-2): 133-135. 
Blacker, D., M. S. Albert, et al. (1994). "Reliability and validity of NINCDS-ADRDA criteria for Alzheimer's disease. The National Institute of Mental Health Genetics Initiative." Arch Neurol 51(12): 1198-204.

Bonne, O., J. M. Gill, et al. (2010). "Corticotropin-releasing factor, interleukin-6, brainderived neurotrophic factor, insulin-like growth factor-1, and substance $\mathrm{P}$ in the cerebrospinal fluid of civilians with posttraumatic stress disorder before and after treatment with paroxetine." J Clin Psychiatry.

Brinton, R. (1993). "Learning and memory." Neuroscience for rehabilitation H. Cohen (edition): 278-279.

Burgener, S., L. Buettner, et al. (2008). "Evidence supporting nutritional interventions for persons in early stage Alzheimer's disease (AD)." The Journal of Nutrition, Health \&amp; Aging 12(1): 18-21.

Cali, A. M. and S. Caprio (2008). "Prediabetes and type 2 diabetes in youth: an emerging epidemic disease?" Current Opinion in Endocrinology, Diabetes and Obesity 15(2): 123-127 10.1097/MED.0b013e3282f57251.

Carrie, I. A. V. K., G; Rolland, Y.; Gillette-Guyonnet, S.; Vellas, B. (2009). "PUFA for Prevention and Treatment of Dementia?" Current Pharmaceutical Design 15: 41734185.

Chin, A. L., S. Negash, et al. (2011). "Diversity and Disparity in Dementia: The Impact of Ethnoracial Differences in Alzheimer Disease." Alzheimer Disease \& Associated Disorders

Cohen, S., D. Janicki-Deverts, et al. (2007). "Psychological stress and disease." Jama 298(14): 1685-7.

Coley, N., S. Andrieu, et al. (2008). "Dementia Prevention: Methodological Explanations for Inconsistent Results." Epidemiologic Reviews 30(1): 35-66.

Collins, T. C., N. J. Petersen, et al. (2003). "The Prevalence of Peripheral Arterial Disease in a Racially Diverse Population." Arch Intern Med 163(12): 1469-1474.

Conrad, C. D. (2006). "What is the functional significance of chronic stress-induced CA3 dendritic retraction within the hippocampus?" Behav Cogn Neurosci Rev 5(1): 41-60.

Cosway, R., M. W. Strachan, et al. (2001). "Cognitive function and information processing in type 2 diabetes." Diabet Med 18(10): 803-10.

Dahl, A., L. B. Hassing, et al. (2010). "Being Overweight in Midlife Is Associated With Lower Cognitive Ability and Steeper Cognitive Decline in Late Life." The Journals of Gerontology Series A: Biological Sciences and Medical Sciences 65A(1): 57-62.

Dangour, A. D., P. J. Whitehouse, et al. (2010). "B-Vitamins and Fatty Acids in the Prevention and Treatment of Alzheimer's Disease and Dementia: A Systematic Review." Journal of Alzheimer's Disease 22(1): 205-224.

Demirovic, J., R. Prineas, et al. (2003). "Prevalence of dementia in three ethnic groups: The south Florida program on aging and health." Annals of Epidemiology 13(6): 472-478.

den Heijer, T., L. J. Launer, et al. (2005). "Association between blood pressure, white matter lesions, and atrophy of the medial temporal lobe." Neurology 64(2): 263-7.

Erickson, K. I., C. A. Raji, et al. (2010). "Physical activity predicts gray matter volume in late adulthood." Neurology 75(16): 1415-1422.

Etgen, T., D. Sander, et al. (2010). "Physical Activity and Incident Cognitive Impairment in Elderly Persons: The INVADE Study." Arch Intern Med 170(2): 186-193.

Everson-Rose, S. A. and T. T. Lewis (2005). "Psychosocial factors and cardiovascular diseases." Annu Rev Public Health 26: 469-500. 
Ferketich, A. K., J. A. Schwartzbaum, et al. (2000). "Depression as an antecedent to heart disease among women and men in the NHANES I study. National Health and Nutrition Examination Survey." Arch Intern Med 160(9): 1261-8.

Fitzpatrick, A. L., L. H. Kuller, et al. (2004). "Incidence and prevalence of dementia in the cardiovascular health study." Journal of the American Geriatrics Society 52(2): 195-204.

Fitzpatrick, A. L., L. H. Kuller, et al. (2009). "Midlife and Late-Life Obesity and the Risk of Dementia: Cardiovascular Health Study." Arch Neurol 66(3): 336-342.

Fontbonne, A., C. Berr, et al. (2001). "Changes in cognitive abilities over a 4-year period are unfavorably affected in elderly diabetic subjects - Results of the Epidemiology of Vascular Aging Study." Diabetes Care 24(2): 366-370.

Gasparini, L. and H. Xu (2003). "Potential roles of insulin and IGF-1 in Alzheimer's disease." Trends in Neurosciences 26(8): 404-406.

Gianaros, P. J., J. R. Jennings, et al. (2007). "Prospective reports of chronic life stress predict decreased grey matter volume in the hippocampus." Neuroimage 35(2): 795-803.

Glass, T. A., K. Bandeen-Roche, et al. (2009). "Neighborhood Psychosocial Hazards and the Association of Cumulative Lead Dose With Cognitive Function in Older Adults." American Journal of Epidemiology 169(6): 683-692.

Gomez-Pinilla, F. (2008). "Brain foods: the effects of nutrients on brain function." Nat Rev Neurosci 9(7): 568-578.

Hackman, D. A. and M. J. Farah (2009). "Socioeconomic status and the developing brain." Trends in Cognitive Sciences 13(2): 65-73.

Harris, M. I., R. Klein, et al. (1992). "Onset of NIDDM occurs at least 4-7 yr before clinical diagnosis." Diabetes Care 15(7): 815-819.

Hillman, C. H., K. I. Erickson, et al. (2008). "Be smart, exercise your heart: exercise effects on brain and cognition." Nat Rev Neurosci 9(1): 58-65.

Hofman, A., A. Ott, et al. (1997). "Atherosclerosis, apolipoprotein E, and prevalence of dementia and Alzheimer's disease in the Rotterdam Study." Lancet 349(9046): 151-4.

Hu, G., C. Sarti, et al. (2005). "The Impact of History of Hypertension and Type 2 Diabetes at Baseline on the Incidence of Stroke and Stroke Mortality." Stroke 36(12): 2538-2543.

Hughes, T., K. Tyler, et al. (2009). "African American caregivers." Dementia 8(1): 95-116.

Hughes, T. F., C.-C. H. Chang, et al. (2010). "Engagement in Reading and Hobbies and Risk of Incident Dementia: The MoVIES Project." American Journal of Alzheimer's Disease and Other Dementias 25(5): 432-438.

Juster, R.-P., B. S. McEwen, et al. (2010). "Allostatic load biomarkers of chronic stress and impact on health and cognition." Neuroscience \& Biobehavioral Reviews 35(1): 2-16.

Kapogiannis, D. and M. P. Mattson (2011). "Disrupted energy metabolism and neuronal circuit dysfunction in cognitive impairment and Alzheimer's disease." The Lancet Neurology 10(2): 187-198.

Khattab, A. D., I. S. Ali, et al. (2005). "Peripheral arterial disease in diabetic patients selected from a primary care setting: Implications for nursing practice." Journal of Vascular Nursing 23(4): 139-148.

Kilander, L., H. Nyman, et al. (1998). "Hypertension Is Related to Cognitive Impairment : A 20-Year Follow-up of 999 Men." Hypertension 31(3): 780-786.

Knopman, D., L. L. Boland, et al. (2001). "Cardiovascular risk factors and cognitive decline in middle-aged adults." Neurology 56(1): 42-48.

Kramer, A. F. and K. I. Erickson (2007). "Capitalizing on cortical plasticity: influence of physical activity on cognition and brain function." Trends in Cognitive Sciences 11(8): 342-348. 
Krieger, N. and S. Sidney (1996). "Racial discrimination and blood pressure: the CARDIA Study of young black and white adults." Am J Public Health 86(10): 1370-8.

Krishnan, S., Y. C. Cozier, et al. (2010). "Socioeconomic Status and Incidence of Type 2 Diabetes: Results From the Black Women's Health Study." American Journal of Epidemiology 171(5): 564-570.

Lan, L. and Z. Lian (2009). "Use of neurobehavioral tests to evaluate the effects of indoor environment quality on productivity." Building and Environment 44(11): 2208-2217.

Lautenschlager, N. T., K. L. Cox, et al. (2008). "Effect of Physical Activity on Cognitive Function in Older Adults at Risk for Alzheimer Disease." JAMA: The Journal of the American Medical Association 300(9): 1027-1037.

Leibson, C. L., J. E. Ransom, et al. (2004). "Peripheral Arterial Disease, Diabetes, and Mortality." Diabetes Care 27(12): 2843-2849.

Logroscino, G., J. H. Kang, et al. (2004). "Prospective study of type 2 diabetes and cognitive decline in women aged 70-81 years." Bmj 328(7439): 548.

Mangiafico, R. A., F. Sarnataro, et al. (2006). "Impaired cognitive performance in asymptomatic peripheral arterial disease: relation to C-reactive protein and Ddimer levels." Age Ageing 35(1): 60-65.

Mangialasche, F., A. Solomon, et al. (2010). "Alzheimer's disease: clinical trials and drug development." The Lancet Neurology 9(7): 702-716.

Manly, J. (2008). "S1-04-03: Alzheimer's disease in the African American population: Implications for genetic studies." Alzheimer's and Dementia 4(4, Supplement 1): T105-T105.

Mattson, M. P., S. Maudsley, et al. (2004). "A neural signaling triumvirate that influences ageing and age-related disease: insulin/IGF-1, BDNF and serotonin." Ageing Research Reviews 3(4): 445-464.

McEwen, B. S. (2008). "Understanding the potency of stressful early life experiences on brain and body function." Metabolism 57(Supplement 2): S11-S15.

McEwen, B. S. and E. Stellar (1993). "Stress and the Individual: Mechanisms Leading to Disease." Arch Intern Med 153(18): 2093-2101.

McKhann, G., D. Drachman, et al. (1984). "Clinical diagnosis of Alzheimer's disease: report of the NINCDS-ADRDA Work Group under the auspices of Department of Health and Human Services Task Force on Alzheimer's Disease." Neurology 34(7): 939-4

Meadows, T. A., D. L. Bhatt, et al. (2009). "Ethnic differences in the prevalence and treatment of cardiovascular risk factors in US outpatients with peripheral arterial disease: insights from the reduction of atherothrombosis for continued health (REACH) registry." Am Heart J 158(6): 1038-45.

Mukherjee, D., K. A. Eagle, et al. (2007). "Impact of Prior Peripheral Arterial Disease and Stroke on Outcomes of Acute Coronary Syndromes and Effect of Evidence-Based Therapies (from the Global Registry of Acute Coronary Events)." The American Journal of Cardiology 100(1): 1-6.

Nakajima, S., I. Ohsawa, et al. (2010). "Regular voluntary exercise cures stress-induced impairment of cognitive function and cell proliferation accompanied by increases in cerebral IGF-1 and GST activity in mice." Behavioural Brain Research 211(2): 178-184.

Nelson, K. M., G. Reiber, et al. (2007). "Peripheral arterial disease in a multiethnic national sample: the role of conventional risk factors and allostatic load." Ethn Dis 17(4): 669-75.

Nielsen, L., T. Seeman, et al. (2007). NIA Exploratory Workshop on Allostatic Load. Washington, DC.

Noble, J. M., J. J. Manly, et al. (2010). "Association of C-Reactive Protein With Cognitive Impairment." Arch Neurol 67(1): 87-92. 
O'Connor, P. J. (2006). "Improving Medication Adherence: Challenges for Physicians, Payers, and Policy Makers." Arch Intern Med 166(17): 1802-1804.

Ott, A., R. P. Stolk, et al. (1996). "Association of diabetes mellitus and dementia: The Rotterdam Study." Diabetologia V39(11): 1392-1397.

Park, D. and A. Gutchess (2006). "The Cognitive Neuroscience of Aging and Culture." Current Directions in Psychological Science 15(3): 105-108.

Peila, R., B. L. Rodriguez, et al. (2002). "Type 2 Diabetes, APOE Gene, and the Risk for Dementia and Related Pathologies: The Honolulu-Asia Aging Study." Diabetes 51(4): 1256-1262.

Phillips, N. A. and C. C. Mate-Kole (1997). "Cognitive Deficits in Peripheral Vascular Disease : A Comparison of Mild Stroke Patients and Normal Control Subjects." Stroke 28(4): 777-784.

Plassman, B. L., K. M. Langa, et al. (2007). "Prevalence of dementia in the united states: The aging, demographics, and memory study." Neuroepidemiology 29(1-2): 125-132.

Rao, R., S. Jackson, et al. (1999). "Neuropsychological Impairment in Stroke, Carotid Stenosis, and Peripheral Vascular Disease : A Comparison With Healthy Community Residents." Stroke 30(10): 2167-2173.

Ritchie, K., S. Artero, et al. (2010). "Caffeine, cognitive functioning, and white matter lesions in the elderly: establishing causality from epidemiological evidence." J Alzheimers Dis 20 Suppl 1: S161-6.

Ritchie, K., I. Carriere, et al. (2010). "Designing prevention programmes to reduce incidence of dementia: prospective cohort study of modifiable risk factors." BMJ 341.

Rose, D. (2010). "Access to Healthy Food: A Key Focus for Research on Domestic Food Insecurity." The Journal of Nutrition 140(6): 1167-1169.

Roth, G. S., J. A. Mattison, et al. (2004). "Aging in Rhesus Monkeys: Relevance to Human Health Interventions." Science 305(5689): 1423-1426.

Rucker-Whitaker, C., P. Greenland, et al. (2004). "Peripheral arterial disease in African Americans: clinical characteristics, leg symptoms, and lower extremity functioning." J Am Geriatr Soc 52(6): 922-30.

Ryan, C. M. and M. O. Geckle (2000). "Circumscribed cognitive dysfunction in middle-aged adults with type 2 diabetes." Diabetes Care 23(10): 1486-93.

Salthouse, T. A. (1996). "The processing-speed theory of adult age differences in cognition." Psychol Rev 103(3): 403-28.

Sapolsky, R. M. (2003). "Stress and plasticity in the limbic system." Neurochem Res 28(11): $1735-42$.

Scarmeas, N., J. A. Luchsinger, et al. (2009). "Physical Activity, Diet, and Risk of Alzheimer Disease." JAMA: The Journal of the American Medical Association 302(6): 627-637.

Selvin, E. and T. P. Erlinger (2004). "Prevalence of and Risk Factors for Peripheral Arterial Disease in the United States: Results From the National Health and Nutrition Examination Survey, 1999-2000." Circulation 110(6): 738-743.

Shoelson, S. E., J. Lee, et al. (2006). "Inflammation and insulin resistance." The Journal of Clinical Investigation 116(7): 1793-1801.

Sieck, G. C. (2003). "Physiology of aging." J Appl Physiol 95(4): 1333-1334.

Singh-Manoux, A., M. Hillsdon, et al. (2005). "Effects of Physical Activity on Cognitive Functioning in Middle Age: Evidence From the Whitehall II Prospective Cohort Study." Am J Public Health 95(12): 2252-2258.

Smith, M. A. (1996). "Hippocampal vulnerability to stress and aging: possible role of neurotrophic factors." Behavioural Brain Research 78(1): 25-36. 
Squadrito, F., G. R. Trimarchi, et al. (1986). "Cerebral cholinergic control of rat arterial blood pressure in streptozotocin-induced diabetes." Pharmacol Res Commun 18(10): 951-65.

Steinberger, J., S. R. Daniels, et al. (2009). "Progress and Challenges in Metabolic Syndrome in Children and Adolescents: A Scientific Statement From the American Heart Association Atherosclerosis, Hypertension, and Obesity in the Young Committee of the Council on Cardiovascular Disease in the Young; Council on Cardiovascular Nursing; and Council on Nutrition, Physical Activity, and Metabolism." Circulation 119(4): 628-647.

Stewart, R. and D. Liolitsa (1999). "Type 2 diabetes mellitus, cognitive impairment and dementia." Diabetic Medicine 16(2): 93-112.

Strachan, M. W., I. J. Deary, et al. (1997). "Is type II diabetes associated with an increased risk of cognitive dysfunction? A critical review of published studies." Diabetes Care 20(3): 438-445.

Sturman, M. T., C. F. M. de Leon, et al. (2008). "Body mass index and cognitive decline in a biracial community population." Neurology 70(5): 360-367.

Taylor, D. H., Jr., F. A. Sloan, et al. (2004). "Marked Increase in Alzheimer's Disease Identified in Medicare Claims Records Between 1991 and 1999." J Gerontol A Biol Sci Med Sci 59(7): M762-766.

van der Beek, E. M. and P. J. G. H. Kamphuis (2008). "The potential role of nutritional components in the management of Alzheimer's Disease." European Journal of Pharmacology 585(1): 197-207.

Van Gerven, P. W. M., H. Vos, et al. (2009). "Annoyance from environmental noise across the lifespan." The Journal of the Acoustical Society of America 126(1): 187-194.

van Hoof, J., H. S. M. Kort, et al. (2010). "The indoor environment and the integrated design of homes for older people with dementia." Building and Environment 45(5): 1244-1261.

Von Dras, D. D. and H. T. Blumenthal (2000). "Biological, Social-Environmental, and Psychological Dialecticism: An Integrated Model of Aging." Basic and Applied Social Psychology 22(3): 199-212.

Waldstein, S. R. (2003). "The relation of hypertension to cognitive function." Current Directions in Psychological Science 12(1): 9-13.

Waldstein, S. R., C. F. Tankard, et al. (2003). "Peripheral Arterial Disease and Cognitive Function." Psychosom Med 65(5): 757-763.

Wang, H.-X., A. Karp, et al. (2002). "Late-Life Engagement in Social and Leisure Activities Is Associated with a Decreased Risk of Dementia: A Longitudinal Study from the Kungsholmen Project." American Journal of Epidemiology 155(12): 1081-1087.

Weiner, M. F. (2008). "Perspective on race and ethnicity in Alzheimer's disease research." Alzheimer's and Dementia 4(4): 233-238.

Welsh, B. and L. Wecker (1991). "Effects of streptozotocin-induced diabetes on acetylcholine metabolism in rat brain." Neurochem Res 16(4): 453-60.

Yaffe, K., D. Barnes, et al. (2001). "A Prospective Study of Physical Activity and Cognitive Decline in Elderly Women: Women Who Walk." Arch Intern Med 161(14): 1703-1708.

Yaffe, K., K. Lindquist, et al. (2003). "Inflammatory markers and cognition in wellfunctioning African-American and white elders." Neurology 61(1): 76-80.

Yan, L. L., K. Liu, et al. (2003). "Psychosocial factors and risk of hypertension: the Coronary Artery Risk Development in Young Adults (CARDIA) study." JAMA 290(16): 2138-48.

Yen, I. H., Y. L. Michael, et al. (2009). "Neighborhood Environment in Studies of Health of Older Adults: A Systematic Review." American Journal of Preventive Medicine 37(5): 455-463. 


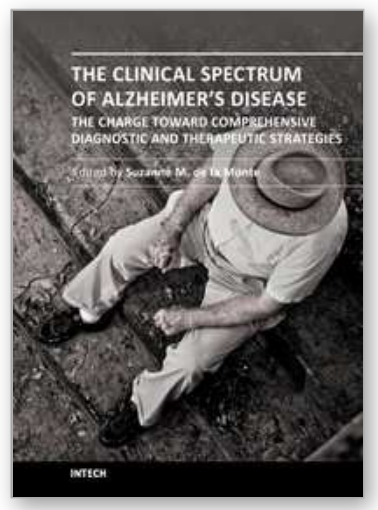

\section{The Clinical Spectrum of Alzheimer's Disease -The Charge Toward Comprehensive Diagnostic and Therapeutic Strategies}

Edited by Dr. Suzanne De La Monte

ISBN 978-953-307-993-6

Hard cover, 362 pages

Publisher InTech

Published online 06, September, 2011

Published in print edition September, 2011

The Clinical Spectrum of Alzheimer's Disease: The Charge Toward Comprehensive Diagnostic and Therapeutic Strategies is highly informative and current. Acknowledged experts in the field critically review both standard and under-appreciated clinical, behavioral, epidemiological, genetic, and neuroimaging attributes of Alzheimer's disease. The collection covers diverse topics of interest to clinicians and researchers alike. Experienced professionals and newcomers to the field will benefit from the read. The strengths and weaknesses of current clinical, non-invasive, neuro-imaging, and biomarker diagnostic approaches are explained. The perspectives give fresh insights into the process of neurodegeneration. Readers will be enlightened by the evidence that the neural circuits damaged by neurodegeneration are much broader than conventionally taught, suggesting that Alzheimer's could be detected at earlier stages of disease by utilizing multi-pronged diagnostic approaches. This book inspires renewed hope that more effective treatments could be developed based upon the expanding list of potential therapeutic targets.

\section{How to reference}

In order to correctly reference this scholarly work, feel free to copy and paste the following:

David L. Mount, Maria Isabel Rego, Alethea Amponsah, Annette Herron, Darin Johnson, Mario Sims, DeMarc Hickson and Sylvia A. Flack (2011). Addressing Risk Factors for Neurocognitive Decline and Alzheimer's Disease Among African Americans in the Era of Health Disparities, The Clinical Spectrum of Alzheimer's Disease -The Charge Toward Comprehensive Diagnostic and Therapeutic Strategies, Dr. Suzanne De La Monte (Ed.), ISBN: 978-953-307-993-6, InTech, Available from: http://www.intechopen.com/books/the-clinicalspectrum-of-alzheimer-s-disease-the-charge-toward-comprehensive-diagnostic-and-therapeuticstrategies/addressing-risk-factors-for-neurocognitive-decline-and-alzheimer-s-disease-among-africanamericans-i

\section{INTECH}

open science | open minds

\section{InTech Europe}

University Campus STeP Ri

Slavka Krautzeka 83/A

51000 Rijeka, Croatia

Phone: +385 (51) 770447

Fax: +385 (51) 686166

\section{InTech China}

Unit 405, Office Block, Hotel Equatorial Shanghai

No.65, Yan An Road (West), Shanghai, 200040, China 中国上海市延安西路65号上海国际贵都大饭店办公楼 405 单元

Phone: +86-21-62489820

Fax: +86-21-62489821 
www.intechopen.com 
(C) 2011 The Author(s). Licensee IntechOpen. This chapter is distributed under the terms of the Creative Commons Attribution-NonCommercialShareAlike-3.0 License, which permits use, distribution and reproduction for non-commercial purposes, provided the original is properly cited and derivative works building on this content are distributed under the same license. 